\title{
A Recommendation System Based on the Education Big Data for E-learning
}

\author{
Ni Lihao ${ }^{12}$ \\ National Engineering Research Center for E-Learning(NERCEL), Central China Normal University, \\ Wuhan, 430079, China \\ E-mail: nilihao20030163.com

\section{Liu Yanshen} \\ Center of Education Information Research, Central China Normal University, Wuhan, 430079, China \\ E-mail: yanshenliu@126.com
}

\section{Liu Yi}

Center of Education Information Research, Central China Normal University, Wuhan, 430079, China E-mail: Iiuyi1360126. com

In order to enable learners to filter the electronic learning materials in the context of education big data, a recommendation system based on the education big data for E-learning is presented in this paper. This paper introduces the basic theory of the membership relations among the learning contents attributes, and establishes the theoretical model of recommendation model based on education big data. The model is built through three steps: setting up the primary fuzzy recommendation demand set, setting up the advanced fuzzy recommendation requirements set and designing a fuzzy recommendation algorithm. And also, this model has designed the framework and functional architecture of the fuzzy recommendation system for learning content. According to the learners' learning situation, the system can select proper learning content and inform learners of the real-time information. The system improves the efficiency of the job selection, and solves the technical problem of the design of the electronic job system in the construction of E-book package. The model is stable, reliable and easy to use.

CENet 2017

22-23 July, 2017

Shanghai, China

\footnotetext{
${ }^{1}$ Correspongding Author

${ }^{2}$ This study is supported by Education Information Research Center of Hubei, Design and implementation of intelligent learning system are based on big data from ( Wenzhou Vocational College of Science and Technology [2015] No.7 ), Research and application of training platform for science and technology competition based on WeChat public number ( the key project of educational technology research program of Zhejiang Province in 2016, JA066 ).

(C) Copyright owned by the author(s) under the terms of the Creative Commons 


\section{Introduction}

Education environment plays an important role in supporting space and external conditions of education activities during the development of education communities[1-2]. Constructing the intelligent learning environment is the core task of wisdom education, including perception, ubiquitous, personalization, integration, predictability and dynamic balance[3].

\section{Related Search}

A lot of education information resources and learning resources are converged in education big data. Chen et, al. argued that the situation of students' learning process should be assessed by the large number of personalized data which are generated in the process of students' learning. Two kinds of recommendation are involved in the traditional way of learning contents: (1)based on learning contents attributes collaborative filtering recommendation, (2)based on learning contents filtering[4-5].

The above methods are generally based on the learning contents, or from the standpoint of teachers. However, the learning situation isoften ignored in these methods, which turns out to be linefficient .

This paper proposesa kind of fuzzy recommendation algorithm from learning contents attribute items, and studies the method of modeling.

\section{Recommendation Model Based on large Data of Education}

\subsection{The Membership Relations Between the Learning Content Attributes}

Learning contents attribute affiliation is the basis of a fuzzy recommendation system. The learning contents attribute is marked with a fuzzy number in the range [0, 1]. To improve the accuracy of the description, each learning contents attribute item is often marked by several different values from different angles [6-7].

The procedure of establishing the relationship of the learning contents which is attributed by the Delphi method is expressed as follows.

$\mathrm{H}=\left\{h_{1}, h_{2}, \ldots, h_{n}\right\}$ is assumed to be the domain of the attribute set of the learning contents $\mathrm{u}, \mathrm{M}$ experts respectively set a value for each attribute item $H_{i}(\mathrm{i}=1,2, \ldots, \mathrm{n})$, the value set by the expert $\mathrm{K}$ is denoted as $\mathrm{S}_{\mathrm{k}}\left(\mathrm{H}_{\mathrm{i}}\right)(\mathrm{i}=1,2, \ldots, \mathrm{n} ; \mathrm{k}=1,2,3 \ldots, \mathrm{m})$, the membership degree of the $\mathrm{M}$ experts is expressed as follows,

$$
S\left(H_{\mathrm{i}}\right)=\frac{1}{m} \sum_{k=1}^{m} S_{k}\left(H_{i}\right)
$$

The learning contents attribute item which is not applicable to expert systems can be expressed as a function based on $\mathrm{H}_{\mathrm{i}}$ as follows,

$$
\mathrm{S}_{\mathrm{f}}\left(H_{i}\right)=F\left(H_{i}\right)
$$

The degree of membership of learning contents of $\mathrm{U}$ can be expressed by $\left\{S^{\prime}\left(H_{i}\right)\right\}$ as follows, 


$$
S^{\prime}\left(H_{i}\right)=\left\{\begin{array}{l}
S\left(H_{i}\right) \ldots \ldots \ldots \ldots \ldots . . H_{i} \\
S_{f}\left(H_{i}\right) \ldots \ldots \ldots \ldots \ldots . . H_{i}
\end{array}\right.
$$

\subsection{Setting up the Primary Fuzzy Recommendation Requirement Set}

According to the characteristics of recommendation, the recommended activity set will reflect the learning interest of the contents from students, which can be used to construct a set of learners' learning requirement.

Set $U=\left\{u_{1}, u_{2}, \ldots, u_{p}\right\}$ as the learner's learning content set, and $u_{1}=\left(S_{11}, S_{12}, \ldots, S_{1 i}, \ldots, S_{1 n}\right)$,

$S 1 i$ is the result of (3), the learning content requirement set can be expressed as follows,

$I=\left\{I_{1}, I_{2}, \ldots, I_{k}, \ldots, I_{p}\right\}, I_{k}$, which represents the primary needs of the learners to study the contents attributes of item $\mathrm{K}$.

$$
I_{k}=\frac{\sum_{j=1}^{p}\left(S_{k}\right)^{2}}{p}
$$

(3.4)expresses the difference of learning content.

\subsection{Setting up the Advanced Fuzzy Recommendation Requirement Set}

Through the analysis of the learning content and learning behavior, we find that the primary fuzzy recommendation requirements set description of the demand for the learner is not clear. Thus, it is necessary to build an advanced fuzzy recommendation requirement sets model.

1. Important attributes

Set $I^{\prime}$ as the expression of the high degree of preference for learning content attributes from learner,and $H=\left\{h_{1}, h_{2}, \ldots, h_{n}\right\}$ is the domain of the item set for learning content $u, M$ experts respectively to set a value for each attribute item $H_{i}(\mathrm{i}=1,2, \ldots, \mathrm{n})$, the value set by the expert $\mathrm{K}$ is denoted as $\mathrm{Q}_{\mathrm{k}}\left(\mathrm{H}_{\mathrm{i}}\right)(\mathrm{i}=1,2, \ldots, \mathrm{n} ; \mathrm{k}=1,2,3 \ldots, \mathrm{m})$, and the membership degree of the $\mathrm{M}$ experts is expressed as follows:

$$
I_{m}\left(H_{i}\right)=\frac{1}{m} \sum_{k=1}^{m} Q_{k}\left(H_{i}\right)
$$

The limitation of the Delphi method is that this method could not be a personalized assignment for each learner. As a result, an improvement for the Delphi method is suggested.

Set $H_{k}=\left\{H_{i}, H_{i}+1, \ldots, H_{i+j}\right\}$, that is, the $H_{k}$ attribute includes the $H_{i} \ldots H_{i+j}$ attribute, recommends the primary demand as $\left\{l_{i}, l_{i+1}, \ldots, l_{i+j}\right\}, l_{i+j}$ represents the primary recommendation requirement of the learning content of item $i+j$.

$$
\text { If } f_{k}=\frac{1}{h} \sum_{h=1}^{j}\left(I_{i+h}-U\right)^{2}
$$

$U$ is the mean of $\boldsymbol{l}_{i+j}$.

2. Advanced recommendation model 
Suppose that a learning content has q attributes, and $I^{\prime}$ is the expression of the high degree of preference, $I_{m}{ }^{\prime}$ indicates the importance of learning content, $I_{f}$ shows the preference dispersion degree of learning content attributes. The three tuple $\left\{I^{\prime}, I_{m}{ }^{\prime}, I_{f}\right\}$ represents the advanced recommendation requirement set. $I=\left\{I^{\prime}, I_{m}{ }^{\prime}, I_{f}\right\}$.

$$
\begin{gathered}
I^{\prime}=\left\{I_{1}^{\prime}, I_{2}^{\prime}, \ldots I_{q}{ }^{\prime}\right\} \\
I_{m}{ }^{\prime}=\left\{I_{m 1}, I_{m 2}, \ldots, I_{m q}\right\} \\
I_{f}=\left\{I_{f 1}, I_{f 2}, \ldots, I_{f q}\right\}
\end{gathered}
$$

\section{Algorithm design}

The learning content recommendation algorithm is proceeded as follows.

if $I_{f}<\alpha 1$ then

$$
S=\left\{A \wedge I^{\prime}\right\} * I_{m}+I_{f}
$$

else if $I_{f}>\alpha 2$ then

\{

$$
\text { if } \begin{aligned}
& A_{k} \not \subset\left\{U_{k}\right\} \\
& \qquad \begin{array}{l}
S=\left\{A \wedge I^{\prime}\right\} * I_{m}+\left(1-I_{f}\right)
\end{array}
\end{aligned}
$$

else $S=\left\{A \wedge I^{\prime}\right\}^{*} I_{m}$

\}

$\alpha 1$ and $\alpha 2$ are two constants, and $\alpha 1<\alpha 2$.

\section{Overall Framework Design of Learning Contents Recommendation System}

\subsection{Overall Framework}

The overall framework of the system is shown in Figure 1.

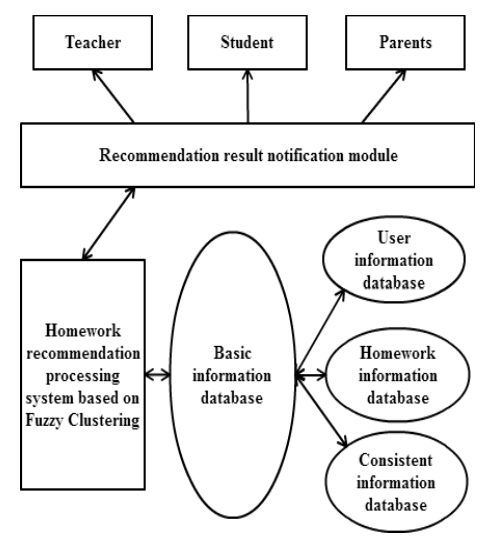

Figure 1: System Frame 


\subsection{Function Architecture}

In this system, the learning content recommendation processing system is the core function module, and the database maintains the learner's learning interest model dynamically. The process of the learning content recommendation processing system is shown in Figure 2. In order to improve the system support for more service providers, the information source can be customized[8].

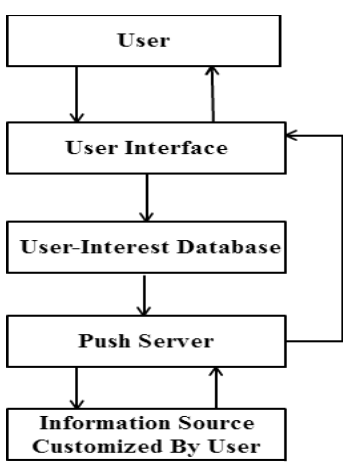

Figure 2: Implementation Process of the Recommended Processing System

\subsection{System Development}

According to the overall framework of the system shown in Figure 1 and the learning content recommended processing flow chart shown in Figure 2, a simulation system is developedas shown in Figure 3.

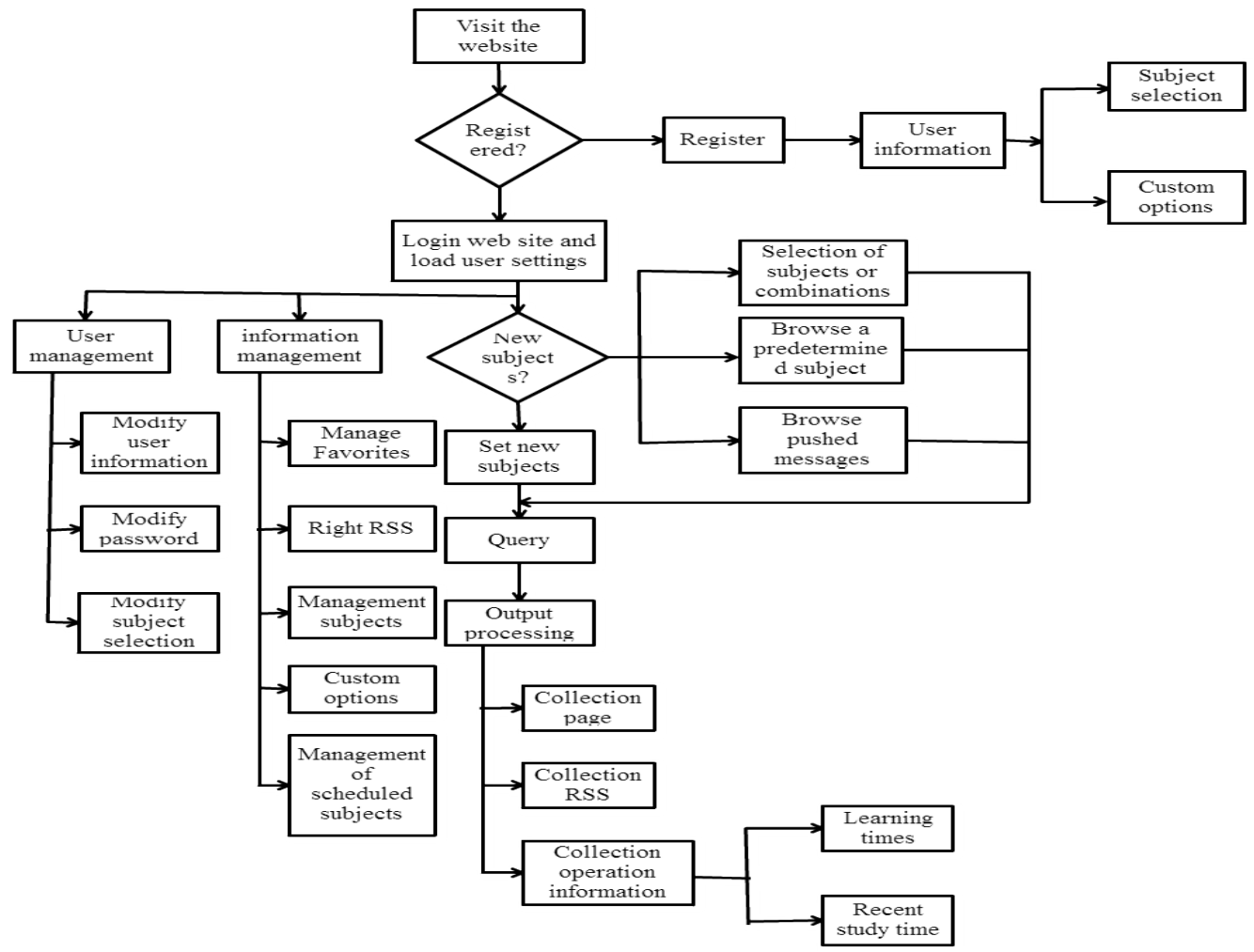

Figure 3: Program Structure

\section{Conclusion}


In the active information recommendation service system, it can be used as the evaluation criteria of information. In our system, a recommen-dation system for these recommended content is provided. Assuming the recommended content that user does not expect is $a 1$, does expect is $a 2$, and expects but not recommend is $a 3$, the sketch of this system is shown in Figure 4.

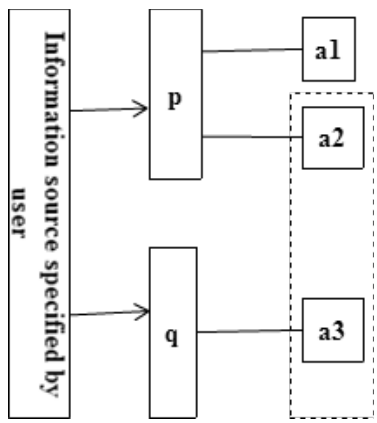

Figure 4: Quantitative Relationship between Push Content and Demand

By running the recommended system model, the data was obtained as shown in Table 1.

\begin{tabular}{lllll}
\hline Interest items & Weight & Push Notifications & Effective & Draw \\
\hline Item1 & 0.15 & 4 & 1 & 4 \\
\hline Item2 & 0.4 & 16 & 13 & 2 \\
\hline Item3 & 0.25 & 6 & 6 & 0 \\
\hline Item4 & 0.2 & 8 & 7 & 0 \\
\hline
\end{tabular}

Table1: Learning Content Recommendation for A User

The usefulness of information is: $\alpha=0.15 \times \frac{1}{1+4}+0.4 \times \frac{13}{13+2}+0.25 \times \frac{6}{6+0}+0.2 \times \frac{7}{7+0} \approx 0.8267$, the percentage of useless information is: $\beta=0.15 \times \frac{3}{3+1}+0.4 \times \frac{3}{3+13}+0.25 \times \frac{0}{0+6}+0.2 \times \frac{1}{1+7} \approx 0.2125$, in extreme cases, the usefulness is: $\chi=0.15 \times \frac{1}{3+1+4}+0.4 \times \frac{13}{3+13+2}+0.25 \times \frac{6}{0+6+0}+0.2 \times \frac{7}{1+7+0} \approx 0.7326$.

With the use of the system, the usefulness of the recommended system will be more and more close to 1 , and the useless will be close to 0 .

\section{References}

[1] IBM: What is big data? www-01.ibm.com/software/data/bigdata. Accessed 26 June 2015.

[2] Chen, C.L.P., Zhang, C.Y., Chen, L., Gan, M.: Fuzzy restricted boltzmann machine for the enhancement of deep learning. IEEE T. Fuzzy Syst. 23(6), 2163-2173 (2015).

[3]Sheng Hong, Zheng Zhou, Enrico Zio, Kan Hong.: Condition assessment for theperformance degradation of bearing based on a combinatorial feature extractionmethod, Digital Signal Processing, 2014, 27:159-166.

[4] Bai, C., \& Sarkis, J. (2013). Green information technology strategic justification and evaluation. Information Systems Frontiers, 15(5). doi:10.1007/s10796-013-9425-x.

[5] Acampora, G., Pedrycz, W., Vitiello, A.: A competent memetic algorithm for learning fuzzy cognitive maps. IEEE T. Fuzzy Syst. 23(6), 2397-2411 (2015). 
[6] Ji, J., Pang, W., Zhou, C., Han, X., Wang, Z.: A fuzzy k-prototype clustering algorithm for mixed numeric and categorical data. Knowledge-Based Syst. 30, 129-135 (2012).

[7] Coletta, L.F., Vendramin, L., Hruschka, E.R., Campello, R.J., Pedrycz, W.: Collaborative fuzzy clustering algorithms: Some refinements and design guidelines. IEEE Trans. Fuzzy Syst. 20(3), 444462 (2012).

[8] Laudon, K., \& Laudon, J. (2010). Management information systems. New Jersey: Prentice Hall. 